\title{
Improving the quality of written information available at weekends in a paediatric hospital: the TRANSMIT sheet
}

\author{
Daniel Hawcutt, Julie Green, Charlotte Durand, Fulya Mehta, Matthew Christopherson, Ian Sinha
}

Alder Hey Children's Hospital

\begin{abstract}
The clinical outcomes at weekends are worse than during the week in a hospital setting. There are many potential factors which influence this. High quality communication between the weekday teams and the on call weekend staff could help improve clinical outcomes at weekends, but there are no validated forms of communication that have been established in a paediatric hospital setting.

The casenotes of all medical patients $(n=119)$ were prospectively evaluated across all medical wards in a large paediatric hospital over three weekends, to establish the quality of information available to on call teams. Following introduction of structured documentation, known as a TRANSMIT (including Tasks, Respiratory, Anticipated problems, Nutrition, Sepsis, Medication, Intravenous access, Transfer/discharge) sheet, the audit was repeated $(n=111)$. A qualitative survey of junior doctors using TRANSMIT was carried out after introduction.
\end{abstract}

Prior to the introduction of the structured documentation (TRANSMIT sheet) an accurate problem list was present in 56\% (67/119), and an adequate written management plan in $63 \%$ (75/119). Following introduction, an improvement in the notes was seen, with accurate problem lists in $82 \%$ (91/111) and an adequate plan in 76\% (84/111). Improvements in the quantity and quality of information available to weekend on call medical staff were noted.

The use of a structured documentation (TRANSMIT sheet) can improve the quality of written information available to on-call teams in a paediatric hospital setting. A retrospective qualitative assessment of junior doctors using TRANSMIT sheets showed an improvement in both the quantity and quality of information available to on call staff at weekends.

\section{Problem}

National bodies are leading a drive to improve the quality of healthcare provided at weekends by NHS hospitals (1). It has long been recognized that clinical outcomes are worse in hospitals during weekends $(2,3)$. This is likely to be due, in part, to the reduced numbers of on-site junior and consultant medical staff, and the fact that on call teams comprise doctors who may not have had adequate training in specialist problems which they cover out-ofhours.

Accurate and comprehensive communication with the on call team is therefore of paramount importance. To ensure that this was occurring in this study's regional children's hospital, which provides primary to quaternary services, the research team evaluated the quality of the casenotes. Given the number of patients, and time constraints, it was implausible that each child would be discussed with the weekend team verbally, but the research team considered it both realistic and reasonable that information in casenotes should be accurate and useful.

The aim of this study, therefore, was to evaluate the quality of information in casenotes, written by the medical teams looking after patients during the week, and then to develop and reassess the changes required to improve the quality of the written information available to the on call team.

\section{Background}

There is good evidence that important information is regularly omitted during handover over in a paediatric environment [4]. Subsequent to this, there has been significant work examining the handover process in paediatric intensive care $[5,6]$, paediatric cardiac surgery [7], and at the interface of primary and secondary care (both admission and discharge) [8] but not across paediatric medical in-patients in general.

This group represents a different challenge to the medical handover process than critical care patients, since the number of patients who are being handed over is significantly higher, and the range of potential problems and plans are potentially greater. There is no published literature that the research team were aware of that deals with the safe and effective handover of this particular paediatric patient population.

\section{Baseline Measurement}

A prospective evaluation of the casenotes of all patients on the medical wards in the hospital was undertaken by members of the study team. The casenotes of 119 medical inpatients were reviewed, over three weekends. Casenotes were examined to see if there was an accurate problem list (compiled within the previous 72 hours), and whether there was a written plan for the weekend team, 
with regards anticipated issues that may arise. Only $67 / 119$ (56\%) casenotes included a recent and accurate problem list, and 75/119 (63\%) had a weekend plan.

See supplementary file: ds2092.pdf - "Figure 1: TRANSMIT sheet used for weekend handover communication"

\section{Design}

The results of the audit were discussed by the study team with medical and nursing staff. Decisions were then made regarding the key data related to patient care that needed to be documented for high quality weekend care, and included Tasks, Respiratory, Anticipated problems, Nutrition, Sepsis, Medication, Intravenous access, Transfer/discharge (TRANSMIT). These were incorporated into the TRANSMIT sheet (Figure 1).

The TRANSMIT sheet is a single page summary to be completed for each patient by the relevant medical team(s) providing care during the week, and filed in the medical notes before the weekend. It replaces the ward round entry in the notes usually taken by the medical team (to ensure that additional workload was not placed on busy clinicians), and provides prompts to the completing team to improve the quality of information handed over. It also contains sections for tasks that will need to be undertaken (e.g. blood tests), and anticipated problems that may occur over the weekend.

Implementation was undertaken following agreement from consultants in the medical specialties, and one-to-one training of fellow junior doctors from all medical teams by the study team. Completion of the TRANSMIT form was undertaken by junior doctors from each medical team on a Friday.

\section{Strategy}

The rationale for this approach was to enable the weekend team to evaluate, immediately, the potential issues for any patient they were asked to see, and to have a recommended plan for certain anticipated problems. To eliminate the duplication of paperwork, the sheet was designed to allow completion as part of the normal ward round process.

\section{Results}

Two months after introducing the TRANSMIT sheets, a re-audit of 111 casenotes, over three weekends, showed that $91 / 111$ (82\%) now included an accurate problem list, and $84 / 111(76 \%)$ had adequate weekend plans. Improvement from before the introduction of the TRANSMIT sheet are shown in Figure 2.

A retrospective qualitative assessment of the TRANSMIT sheets was obtained from questionnaires to medical staff, using a 1-6 scale ( $1=$ strongly disagree, $6=$ strongly agree) and was overwhelmingly positive. Improvements were noted in:

1. The efficiency of weekend ward rounds (pre TRANSMIT median score 1 [25th-75th centiles 1-2], post TRANSMIT median score 3.5 [25th-75th centile 2-4])

2. The understanding of patients problems by weekend teams (pre TRANSMIT median score 4 [25th-75th centiles 2-4], post TRANSMIT median score 5 [25th-75th centiles 3-5] .

3. Weekend plan containing enough information to make safe and appropriate decisions (pre TRANSMIT median score 3 [25th-75th centiles 2-4], post TRANSMIT median score 4 [25th-75th centiles 2.5-5])

In addition, junior doctors felt that TRANSMIT sheets improved safety out of hours (median score 5.5 [25th-75th centiles 5-6])

The TRANSMIT forms are still routinely completed by all medical teams one year on from initiating this project.

See supplementary file: ds2172.pdf - "Figure 2: Effects of implementation of TRANSMIT sheets"

\section{Lessons and Limitations}

Several lessons have been learnt by the implementation of TRANSMIT sheets.

Firstly, the introduction of a structured form of written communication between specialty paediatric services and the weekend on call team in our hospital improved the quantity and quality of information for treatment. A TRANSMIT sheet (or adaptation) is therefore potentially utilizable in other settings which provide paediatric in-patient care.

Secondly, the nature of any structured written communication needs to be simple and quick to complete. The TRANSMIT sheet was developed by junior medical staff, in collaboration with nursing staff and consultants, and this has helped foster a feeling of collective ownership and ensured that the included items are usefu and appropriate. Completion of the sheet as part of normal ward round paperwork minimised the impact on busy teams. These reasons have probably contributed to the good utilization and impact of the TRANSMIT sheet.

The main barrier to its effective use is that the quality of the information provided on the form is variable, and this is an issue the study team aim to prospectively evaluate. The team also aim to develop a better training programme around handover of patients, and to incorporate TRANSMIT sheets within this.

With regards to the limitations of the study's audit, the team have only evaluated two key components of written handover (problem list and weekend plan). Because of the infrequent nature of serious safety events in children, an assessment of whether utilizing the TRANSMIT sheet has improved patient safety was outside the scope of the project.

\section{Conclusion}

Use of a structured documentation (TRANSMIT sheet) can improve the quality of written information available to on call teams in a 
paediatric hospital setting. A retrospective qualitative assessment of junior doctors using TRANSMIT sheets showed an improvement in both the quantity and the quality of information available to on call staff at weekends.

\section{References}

1. Keogh B. Should the NHS work at weekends as it does in the week? Yes. BMJ 2013;20:f621.

2. Bell CM, Redelmeier DA. Mortality among patients admitted to hospitals on weekends as compared with weekdays. NEJM 2001;345:663-8.

3. Aylin P, Yunus A, Bottle A, Majeed A, Bell D. Weekend mortality for emergency admissions. A large, multicentre study. Qual Saf Health Care 2010;19:213-7.

4. Borowitz, SM, Waggoner-Fountain, LA, Bass, EJ, Sledd, RM. Adequacy of information transferred at resident signout (inhospital handover of care): a prospective survey Qual Saf Health Care 2008;17:6-10.

5. Agarwal, Hemant S, Saville, Benjamin R, Slayton, Jennifer $\mathrm{M}$, et al. Standardized postoperative handover process improves outcomes in the intensive care unit: A model for operational sustainability and improved team performance. Crit Care Medicine 2012; 40(7): 2109-2115.

6. Craig, Rona, Moxey, Linda, Young, David, et al. Strengthening handover communication in pediatric cardiac intensive care. PEDIATRIC ANESTHESIA 2012; 22(4): 393-399.

7. Zavalkoff, Samara R., Razack, Saleem I, Lavoie, Josee, et al. Handover after pediatric heart surgery: A simple tool improves information exchange. PEDIATRIC CRITICAL CARE MEDICINE 2011; 12(3); 309-313.

8. HANDOVER: Improving the Continuity of Patient Care Through Identification and Implementation of Novel Patient Handover Processes in Europe

http://www.handover.eu/project.html accessed 10.09.13

\section{Declaration of interests}

No competing interests to declare

\section{Acknowledgements}

We would like to thank the Nurses and Doctors at Alder Hey Children's Hospital who contributed to the design and successful implementation of this work across the hospital. 